\title{
Manual therapy compared with physical therapy in patients with non-specific neck pain: a randomized controlled trial
}

\author{
Ruud Groeneweg ${ }^{1,2,3^{*}}$, Luite van Assen ${ }^{1}$, Hans Kropman ${ }^{3}$, Huco Leopold ${ }^{3}$, Jan Mulder ${ }^{1}$,
}

Bouwien C. M. Smits-Engelsman ${ }^{4}$, Raymond W J. G. Ostelo ${ }^{2,5}$, Rob A. B. Oostendorp ${ }^{1,6}$ and Maurits W. van Tulder ${ }^{2}$

\begin{abstract}
Background: Manual therapy according to the School of Manual Therapy Utrecht (MTU) is a specific type of passive manual joint mobilization. MTU has not yet been systematically compared to other manual therapies and physical therapy. In this study the effectiveness of MTU is compared to physical therapy, particularly active exercise therapy (PT) in patients with non-specific neck pain.

Methods: Patients neck pain, aged between 18-70 years, were included in a pragmatic randomized controlled trial with a one-year follow-up. Primary outcome measures were global perceived effect and functioning (Neck Disability Index), the secondary outcome was pain intensity (Numeric Rating Scale for Pain). Outcomes were measured at 3, 7, 13, 26 and 52 weeks. Multilevel analyses (intention-to-treat) were the primary analyses for overall between-group differences. Additional to the primary and secondary outcomes the number of treatment sessions of the MTU group and PT group was analyzed. Data were collected from September 2008 to February 2011.
\end{abstract}

Results: A total of 181 patients were included. Multilevel analyses showed no statistically significant overall differences at one year between the MTU and PT groups on any of the primary and secondary outcomes. The MTU group showed significantly lower treatment sessions compared to the PT group (respectively 3.1 vs. 5.9 after 7 weeks; 6.1 vs. 10.0 after 52 weeks).

Conclusions: Patients with neck pain improved in both groups without statistical significantly or clinically relevant differences between the MTU and PT groups during one-year follow-up.

Trial registration: ClinicalTrials.gov Identifier: NCT00713843.

Keywords: Randomized controlled trial, Neck pain, Manual therapy, Physical therapy, Effectiveness

\section{Background}

In terms of number of years lived with disability, back and neck pain are most important condition worldwide [1] and usually runs an episodic course over a person's lifetime [2-4]. Neck pain is an important individual, social and economic health problem, affecting up to twothirds of adults at some point in their lives [3]. In the

\footnotetext{
* Correspondence: r.groeneweg@vu.nl

'Radboud University Nijmegen Medical Centre, Scientific Institute for Quality of Health Care, Geert Grooteplein 21, 6525 EX Nijmegen, The Netherlands

${ }^{2}$ Department of Health Sciences, Faculty of Earth \& Life Sciences, Vrije

Universiteit Amsterdam, Amsterdam Public Health research institute, De

Boelelaan 1085, 1081 HV Amsterdam, The Netherlands

Full list of author information is available at the end of the article
}

Netherlands, neck pain is the third most frequently selfreported musculoskeletal pain problem [5], Although many neck pain sufferers do not consult a health professional [6], the prevalence and costs of neck pain in primary care are high $[7,8]$. Furthermore, neck pain has a major effect on participation, activities, work disabilities and is consequently associated with high indirect costs $[3,5,9]$.

In cases of acute neck pain, general practitioners (GP) often take no immediate action [10]. If complaints persist for six months or longer, average perceived discomfort appears to remain fairly stable [11]. However, it is both clinically and economically important to ensure that patients do not enter a chronic phase. Evidence 
regarding treatment efficacy for sub-acute and chronic neck pain is currently inconclusive [12].

Manual therapy is a commonly used treatment for neck pain. Cochrane Reviews have shown that both manual therapy [12] and exercise therapy [13] are effective in the treatment of patients with neck pain. Studies in the Netherlands [14-16] in patients with sub-acute and chronic neck pain has shown significant differences in effectiveness and cost-effectiveness in favor of manual therapy compared with exercise therapy or usual GP care, both in short and long-term follow-up. Manual therapy according to the School of Manual Therapy Utrecht (MTU) is a specific form of spinal manipulative therapy in the Netherlands that differs in theoretical assumptions and principles from other manual therapies $[17,18]$. In general, most manual therapies focus primarily on patient's symptoms, particularly the main complaint, and on joint function and stability, range of movement, and the severity of symptoms [18]. MTU, in contrast, is guided by an assessment of preferred movement patterns of the individual patient and is performed by applying passive articular movements to all spinal and pelvic joints and all joints of extremities, with the goal of optimizing individual movement patterns. Treatment techniques used in MTU are based on arthrokinematic and osteokinematic principles and are comparable with the mobilization techniques used in other manual therapies. The main difference between MTU and other manual therapies and physical therapy is the assessment and treatment of the complete chain of joints of the spine, pelvis and extremities, independently of patient's complaints, based on analysis of the individual movement pattern. Examples of preferred movement patterns are hand clasping, arm folding, and dominance of arm, leg and eye.

To date, no study has evaluated the effectiveness of MTU. Therefore, the objective of this study was to compare the short-term (7 weeks) and long-term effectiveness (52 weeks) of passive mobilization of the joint chain per MTU with active exercise therapy as usual care for physical therapy. The assumption was that MTU was more effective because of the above-mentioned characteristics of MTU. Patients with neck pain (more than two weeks and no longer than one year) were assessed regarding global perceived effect, functioning and pain.

\section{Methods}

Methods have been described in detail elsewhere, and will be summarized below [17].

\section{Design overview}

A pragmatic randomized controlled trial (RCT) was performed from September 2008 to February 2011.

\section{Settings and participants}

Sixteen primary care practices participated in the trial. The manual therapists were enrolled via invitation by the research team and by collective invitation to the members of the professional association of the School of MTU. Participating manual therapists were asked to invite physical therapists (performing the active exercise therapy) with whom they collaborate in this study. Each participating practice had at least one manual therapist, one physical therapist and one research assistant. The latter were trained to perform the intake in a face-to-face meeting and with a video application. Twenty research assistants performed clinical tests to assess inclusion and exclusion criteria, and carried out history taking at baseline. Seventeen manual therapists and 27 physical therapists were involved in the study. Manual therapists had followed a three-year postgraduate program at the School of MTU following their physical therapy education. All participating therapists had a minimum of five years' work experience.

Inclusion criteria for patient participation were: age 18 to 70 years; neck pain of any severity (cervical region from superior nuchal line to spine of scapula and superior border of the clavicula [4]) with or without radiation to the shoulder region or the upper extremity, with or without headaches; neck pain as main complaint for more than two weeks [4] and no longer than one year (inclusion of patients without spontaneous recovery within two weeks and without typical characteristics of long-lasting chronic neck pain, because these groups may benefit less from manual therapy); provocation or reproduction of pain by neck movement or neck and head posture.

Exclusion criteria were: presence of red flags, myelopathy, surgery of the cervical spine [19]; neck pain with a radicular pain pattern; entrapment neuropathy; pregnancy; whiplash injury (as cause of the complaint); any physical treatment for neck pain in the previous three months.

Patients entered the study either through direct access to a primary care practice or by GP referral, according to the Dutch healthcare system. Some patients were also recruited through articles in local newspapers. After signing informed consent, randomization took place.

\section{Randomization and interventions}

Block randomization (block size of 4) was performed, prestratified for main complaint on numeric rating scale for pain (NRS-P) (range 0 (no pain) -10 (maximum pain)) $(<7$ or $\geq 7)$ and age ( $<40$ or $\geq 40$ years) [20, 21]. Independent research assistants, blinded for patient characteristics, allocated patients to one of the intervention groups using a central computer-generated randomization scheme. 


\section{Manual therapy}

MTU assessment is based on theoretical concepts of mechanobiology [22], and is described in the design article of this study [17]. General assessment includes history taking, screening for red flags, physical examination and treatment indication by the manual therapist. In addition, specific tests are used to evaluate the individual's preferred movement pattern [23]. Examples of preferred movement patterns are hand clasping, arm folding, and dominance of arm, leg and eye. These tests have a high reliability (Kappa 0.8-1) [24].

The complete chain of joints of the spine, pelvis and extremities are mobilized whereby the direction of mobilization is theoretically determined by analysis of the tests of the individual preferred movement patterns. The manual therapist performs per protocol repeated passive joint movements with low velocity and intensity and high accuracy in different positions of the patient (sitting, supine and side-lying). The rhythm of the movements is slow (approximately 30 cycles $/ \mathrm{min}$ ) and the movements are repeated about six times. Treatment is in general painless. Passive joint movements are performed in a combination of rolling and sliding, or rocking and gliding (or swinging and sliding) in the joint, based on the arthrokinematic and osteokinematic principles of intra-articular movements. Passive movements are performed over the entire range of motion within the physiological range of motion of joints, whereby the curvature of the articular surface is followed, with manual forces directed to the joints/specific spinal level. Physiological joint range of motion is carefully respected. Traction, oscillation and high-velocity movements are not applied. In all patients, based on the assessment protocols, all joints of the spine, pelvis and extremities are mobilized in specific directions. The kind of the joint mobilization used is probably best comparable to grade III mobilization according to the principles of Maitland [25]. It is common to give advice on activities of daily living and lifestyle, and to recommend home exercises, customized to the patient as assessed by the manual therapist.

A treatment session takes 30 to $60 \mathrm{~min}$. Treatment is repeated after one or two weeks. The maximum number of sessions is six over a six-week treatment period, determined per patient by the manual therapist and depending on the condition of the patient and/or progression of patient's condition.

\section{Physical therapy}

Prior to patient enrollment the participating physical therapists met and reached consensus on the treatment protocol. At the first appointment, the physical therapist enquired about history taking and physical examination. Treatments could consist of active exercise therapy, manual traction, muscle stretching and massage [26-28]. Manual mobilization techniques of the neck were not allowed. The aim of active exercises was to improve strength (particularly strengthening of the deep neck muscles and shoulder muscles), mobility of the neck, and movement coordination. Tailoring treatment was left to the discretion of the therapists and was based on a patient's individual abilities, tolerance, condition and activities of daily living. The intensity level of the treatment was not defined.

Treatment sessions took place no more than twice a week, with a maximum of nine sessions. Session duration was approximately $30 \mathrm{~min}$, determined per patient by the therapist. In each session, the physical therapist spent a minimum of twenty minutes on active exercise therapy combined with instruction. Advice on activities of daily living and lifestyle was also commonly offered to patients.

All participating manual therapists and physical therapists received a three-hour instruction session regarding interpretation and application of the study protocol. Deviations from the study protocol were registered, as were continuation of the treatment and co-interventions.

\section{Co-interventions}

During the intervention period, participating patients were asked not to use any other treatment besides those allocated, except for medication. Patients were free to use medication prescribed either by a physician or overthe-counter.

\section{Outcomes and follow-up}

The patients completed questionnaires online or as a hard copy at baseline, 3, 7, 13, 26 and 52 weeks [17].

\section{Prognostic factors}

At baseline, demographic data, complaints and known prognostic factors were checked by history taking and questionnaires (Table 1). The Credibility/Expectancy Questionnaire (CEQ) [29] was completed, on a hard copy blinded for the therapist, after the first treatment session because expectations can influence treatment outcomes $[30,31]$. The CEQ has been shown to be sufficiently valid and reliable [30]. Fear avoidance, measured with the Fear Avoidance Belief Questionnaire (FABQ) [32], is a risk factor for chronic pain and disability $[31,33,34]$ and can predict outcomes $[35,36]$. The general health questionnaire (SF36) was used to obtain a detailed health profile. The eight domains of the SF36 can be summarized into physical and mental component scores. The Dutch translation showed satisfactory validity and reliability ( 0.66 to 0.90 , mean 0.84) [37]. Prior to randomization at baseline the research assistant verbally asked patients, and notes, if they had preferences to be treated with MTU or PT. 
Table 1 Baseline characteristics of the MTU group $(n=90)$ and PT group $(n=91)$

\begin{tabular}{lll}
\hline Variable & MTU $(n=90)$ & PT $(n=91)$ \\
\hline Age (in years, mean; SD) & $49.2(12.4)$ & $48.7(12.6)$ \\
Gender female (n; \%) & $56(62.2)$ & $56(61.5)$ \\
First time neck complaints (n; \%) & $56(62.2)$ & $58(63.7)$ \\
3 or more musculoskeletal complaints & $54(60.0)$ & $55(59.8)$ \\
4 or more musculoskeletal complaints & $27(30.0)$ & $26(28.3)$ \\
Combined neck and back pain & $15(16.7)$ & $16(17.4)$ \\
Last Year visit GP for neck complaints (n; \%) & $23(25.6)$ & $26(28.6)$ \\
Last Year visit any therapist for neck (n; \%) & $17(18.9)$ & $20(22.0)$ \\
Main complaints (n; \%) & & \\
$\quad$ Pain & $79(87.8)$ & $80(87.9)$ \\
$\quad$ Stiffness & $30(33.3)$ & $38(41.8)$ \\
$\quad$ Mobility impairment & $33(36.7)$ & $37(40.7)$ \\
$\quad$ Other & $7(7.8)$ & $10(11.0)$ \\
Complain intensity (NRS 0-10) & & \\
$\quad$ Main complaint (mean; SD) & $6.9(1.3)$ & $6.8(1.4)$ \\
Second complaint (mean; SD) & $6.0(1.6)$ & $5.9(1.8)$ \\
Third complaint (mean; SD) & $5.1(2.1)$ & $5.3(2.0)$
\end{tabular}

NDI (0-50) (mean; SD)

$12.5(6.8) \quad 11.7(5.4)$

NRS pain (0-10) (mean; SD)

$\begin{array}{lll}\text { FABQ (mean; SD) } & & \\ \text { Total (0-96) } & 30.0(16.4) & 29.6(15.1) \\ \text { Subscale work (0-66) } & 14.7(13.5) & 14.8(12.3) \\ \text { Subscale physical activity (0-30) } & 15.3(5.4) & 14.8(5.7) \\ \text { SF-36 (mean; SD) } & & \\ \text { Physical component summary (0-100) } & 44.6(7.9) & 44.6(7.1) \\ \text { Mental component summary (0-100) } & 46.4(12.5) & 47.2(10.0) \\ \text { CEQ (mean; SD) } & & \\ \text { Credibility (0-27) } & 22.0(3.4) & 22.4(3.2) \\ \text { Expectancy (0-27) } & 21.9(4.8) & 22.1(4.7) \\ \text { Intervention preference ( } n \text {; \%) } & & \\ \text { None } & 61(67.8) & 63(69.2) \\ \text { Manual therapy } & 20(22.2) & 20(22.0) \\ \text { Physical therapy } & 9(10.0) & 8(8.8) \\ \text { Pre-stratification } & & 6 \\ \text { A. NRS main complaint } \leq 6, \text { age } \leq 39 & 5 & 23 \\ \text { B. NRS main complaint } \leq 6 \text {, age } \geq 40 & 23 \\ \text { C. NRS main complaint } \geq 7 \text {, age } \leq 39 & 16 & 16 \\ \text { D. NRS main complaint } \geq 7 \text {, age } \geq 40 & 46 & 46\end{array}$

Abbreviations: MTU Manual Therapy Utrecht, PT Physical Therapy, SD Standard Deviation, NRS pain Numeric Rating Scale, NDI Neck Disability Index, FABQ Fear Avoidance Belief Questionnaire, SF-36 Short Form-36, CEQ Credibility/Expectancy Questionnaire, SD Standard deviation

\section{Primary outcomes}

Global perceived effect (GPE) was measured using a 7point ordinal scale (ranging from 'much worse' (1 point) to 'complete recovery' (7 points)). The GPE was dichotomized in responders (patients with 'complete recovery' (7 points) and 'much improved' (6 points)) and nonresponders ('slightly improved,' 'no change,' slightly worse', 'much worse', 'worse than ever'). The GPE measures patient subjective global improvement and has a high face validity $[38,39]$ and an excellent test-retest reliability (ICC values of 0.90 to 0.99 ) [40].

Functioning was measured using the Neck Disability Index (NDI) [41]. The range of scores is 0-50; the higher the score, the greater the limitations in activities. The validity of the NDI is good [41], and reproducibility $[42,43]$ and responsiveness are acceptable [42-44]. The minimal clinically-important change (MCIC) on the NDI is 3.5 points $[42,44]$.

\section{Secondary outcomes}

Secondary outcomes were chosen to cover all domains of the International Classification of Functioning, Disability and Health (ICF) [45]. The NRS-P (range $0=$ no pain to $10=$ maximum pain) was used to assess neck pain intensity in the previous week. The NRS is a valid and responsive scale [46]. The MCIC on the NRS-P in patients with neck pain is 2 points [47].

Patients also registered severity, time of onset, duration and type of adverse events, when relevant, at 3, 7 and 13 weeks [48].

Additional the number of treatment sessions of the MTU group and PT group was analyzed.

\section{Sample size}

Sample size calculation was based on both primary outcomes (GPE and NDI), whereby the GPE was chosen because this outcome variable requires the largest group of participants. A clinically-relevant difference of $20 \%$ in GPE was chosen, based on previous studies [14, 49]. Based on an $\alpha$ of .05 and $80 \%$ power $(\beta=0.2)$, 76 participants per intervention group were required. Considering a dropout rate of $15 \%$, the aim was to include 90 participants in each group.

\section{Statistical analysis}

The analyses were performed according to the intentionto-treat principle. A multilevel model was used to determine the effectiveness of the interventions over the follow-up period. In the analysis, patients were nested by practices Linear multilevel analyses were used for continuous variables and logistic multilevel analyses for dichotomous variables. All patients were analyzed. In multilevel analysis missing scores do not need an imputation strategy, as this type of analysis is very flexible in 
handling missing data [50]. As a secondary analysis, and following the intended analysis described in our design article [17], the differences between groups were tested by the Chi-Square test for dichotomous variables, and by ANOVA/mixed model (continuous variables), with Bonferroni post-hoc tests. A responder analysis was carried out on GPE and the NDI with a MCIC of $\geq 3.5$ points [42] and for NRS-P on $\geq 2$ points [47].

For evaluation of adverse events and patient preferences, Chi-square tests for categorical data and ANOVA, with Bonferroni post-hoc tests were used for continuous data. An assistant, blinded for patient details, handled all data registration. SPSS statistical software version 23 was used. For all comparisons, $P \leq 0.05$ was considered statistically significant (two-sided). Missing values less than $15 \%$ were considered as acceptable, as suggested by PEDRO [51].

\section{Results}

\section{Patient characteristics and baseline similarities}

A total of 221 patients were eligible, of whom 181 were randomized, ranging from 2-41 over the practices (see flow chart, Fig. 1, for more details). Mean patient age was 49 years $(\mathrm{SD}=12.5)$, and approximately $55 \%$ were women. Most patients (57\%) had neck complaints for the first time and most patients had multiple musculoskeletal complaints $(60 \%)$. There were no important differences at baseline between the groups (Table 1).

\section{(Intention-to-treat) analysis}

Missing values accounted for $3.9 \%$ at 7 weeks and $8.3 \%$ at 52 weeks. In multilevel analyses, there were no statistically significant overall differences at one year between the two groups on the primary and secondary outcomes GPE, NDI, or NRS-P (Table 2, Fig. 2). GPE showed statistically significantly higher scores only at 3 weeks in favor of the MTU group; adjusted odds ratio 3.97 (95\% confidence interval $[\mathrm{CI}], 1.31$ to $12.04 ; P=0.02$ ). Pain intensity scores were lower for the PT group only at 26 weeks; mean adjusted differences of 0.78 points ( $95 \%$ CI, 0.09 to $1.47,95 \%$ CI; $P=0.03$ ). There were no other statistically significant differences. ANOVA for NDI and NRS-P, and Chi-square tests for GPE, showed identical results.

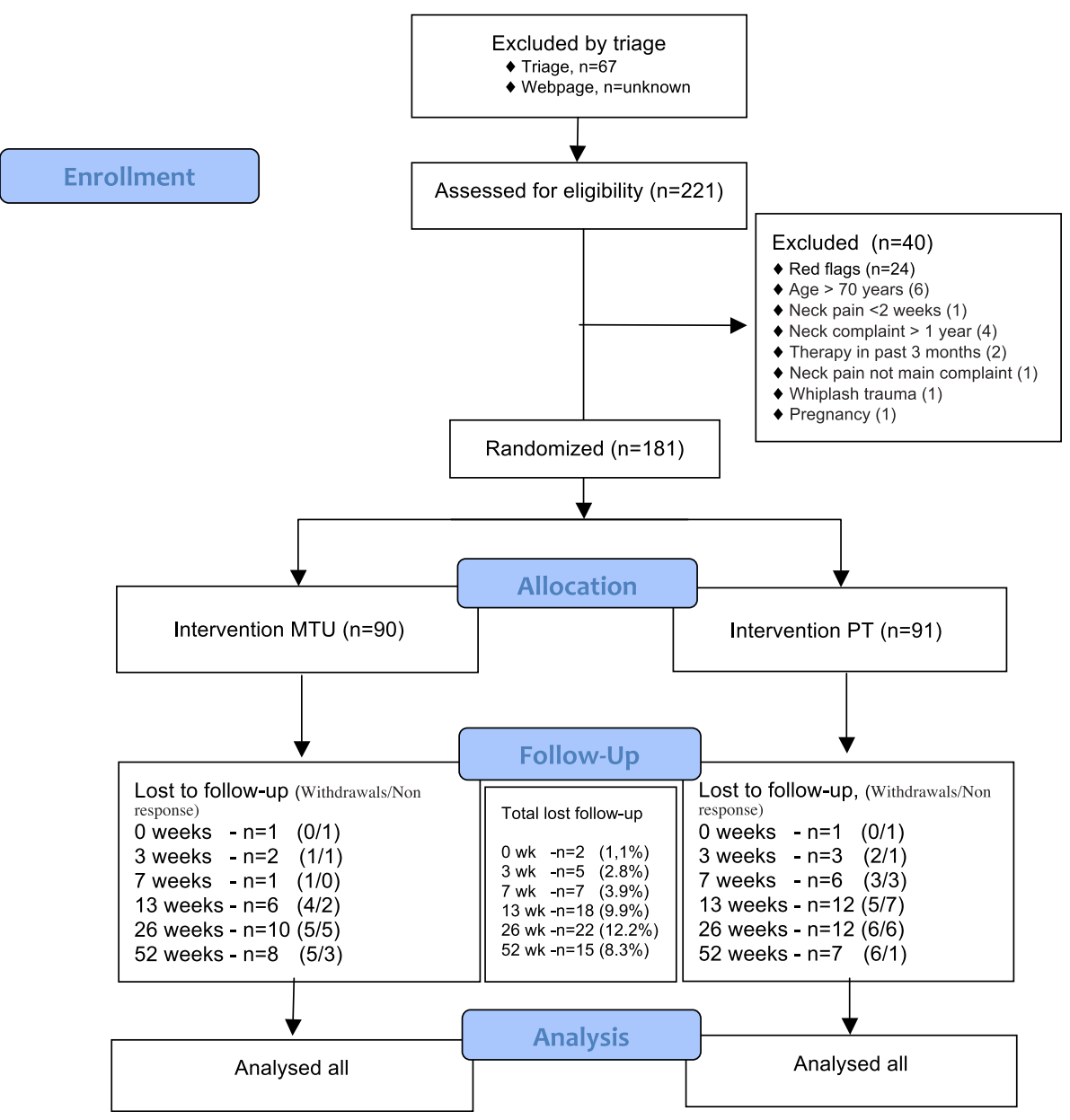

Fig. 1 Flowchart of participation in the NECK project trial 
Table 2 Mean scores, regression coefficients, and odds ratios for primary and secondary outcomes, and responder analyses (Intention to Treat, $N=181$ )

\begin{tabular}{|c|c|c|c|c|}
\hline Variables & $\begin{array}{l}\text { Manual therapy } \\
\text { Utrecht }(n=90)\end{array}$ & $\begin{array}{l}\text { Physiotherapy } \\
(n=91)\end{array}$ & $\begin{array}{l}\text { Regression coefficients } \\
(95 \% \mathrm{Cl})\end{array}$ & \\
\hline \multicolumn{5}{|c|}{ Primary outcomes measures } \\
\hline GPE (success, \%) & & & OR $(95 \% \mathrm{Cl})$ & P \\
\hline \multicolumn{5}{|l|}{ Overall effect: $P=0.20$} \\
\hline 3 weeks. & 30.7 & 14.8 & 3.97 (1.31 to 12.04$)$ & $0.02^{* *}$ \\
\hline 7 weeks. & 51.7 & 42.4 & 1.900 .69 to 5.20$)$ & 0.21 \\
\hline 13 weeks. & 60.7 & 57.0 & $1.11(0.39$ to 3.15$)$ & 0.84 \\
\hline 26 weeks. & 57.5 & 59.5 & 0.76 (0.26 to 2.18$)$ & 0.61 \\
\hline 52 weeks. & 64.9 & 56.0 & 1.72 (0.61 to 4.88 ) & 0.31 \\
\hline $\mathrm{NDI}(0-50)(\mathrm{M}, \mathrm{SD})$ & & & $\begin{array}{l}\text { Regression Coefficients } \\
(95 \% \mathrm{Cl})\end{array}$ & $P$ \\
\hline \multicolumn{5}{|l|}{ Overall effect: $P=0.57$} \\
\hline Baseline & $12.5(6.8)$ & $11.7(5.4)$ & & \\
\hline 3 weeks. & $10.5(6.9)$ & $10.2(5.6)$ & $-0.24(-1.65$ to 1.16$)$ & 0.74 \\
\hline 7 weeks. & $7.8(6.4)$ & $8.1(7.1)$ & $-0.69(-2.14$ to 0.76$)$ & 0.35 \\
\hline 13 weeks. & $5.9(5.8)$ & $5.9(4.6)$ & $-0.23(-1.67$ to 1.21$)$ & 0.75 \\
\hline 26 weeks. & $5.9(5.5)$ & $5.6(5.6)$ & $0.34(-1.11$ to 1.80$)$ & 0.64 \\
\hline 52 weeks. & $5.9(5.7)$ & $6.6(6.5)$ & $-0.82(-2.25$ to 0.61$)$ & 0.26 \\
\hline \multicolumn{5}{|c|}{ Secondary outcomes measures } \\
\hline \multicolumn{5}{|l|}{ NRS-P $(0-10)(M, S D)$} \\
\hline \multicolumn{5}{|l|}{ Overall effect: $P=0.76$} \\
\hline Baseline & $5.5(2.0)$ & $5.8(1.8)$ & & \\
\hline 3 weeks. & $4.2(2.3)$ & $4.6(2.1)$ & $-0.27(-0.94$ to 0.40$)$ & 0.42 \\
\hline 7 weeks. & $3.2(2.3)$ & $3.6(2.4)$ & $-0.20(-0.87$ to 0.47$)$ & 0.56 \\
\hline 13 weeks. & $2.8(2.5)$ & $2.6(2.0)$ & $0.24(-0.44$ to 0.93$)$ & 0.49 \\
\hline 26 weeks. & $2.9(2.4)$ & $2.3(2.2)$ & 0.78 (0.09 to 1.47$)$ & $0.03^{* *}$ \\
\hline 52 weeks. & $2.5(2.6)$ & $2.8(2.6)$ & $-1.14(-0.82$ to 0.54$)$ & 0.69 \\
\hline \multicolumn{5}{|l|}{ Responder analyses } \\
\hline MCID NDI (success, \%) & & & OR (95\% Cl) & P \\
\hline \multicolumn{5}{|l|}{ Overall effect: $P=0.95$} \\
\hline 3 weeks. & 31.0 & 20.7 & 2.08 (0.79 to 5.43$)$ & 0.14 \\
\hline 7 weeks. & 56.3 & 52.0 & 1.26 (0.49 to 3.24$)$ & 0.63 \\
\hline 13 weeks. & 66.3 & 65.8 & 0.97 (0.37 to 2.53$)$ & 0.95 \\
\hline 26 weeks. & 62.0 & 69.6 & $0.55(0.21$ to 1.47$)$ & 0.23 \\
\hline 52 weeks. & 60.5 & 63.1 & $0,79(0.31$ to 2.02$)$ & 0.63 \\
\hline \multicolumn{5}{|l|}{ MCID NRS-P (success, \%) } \\
\hline \multicolumn{5}{|l|}{ Overall effect: $P=0.11$} \\
\hline 3 weeks. & 34.9 & 37.2 & 0.87 (0.38 to 2.14$)$ & 0.73 \\
\hline 7 weeks. & 55.2 & 56.6 & 0.95 (0.42 to 2.14$)$ & 0.89 \\
\hline 13 weeks. & 59.4 & 65.2 & $0.56(0.23$ to 1.38$)$ & 0.21 \\
\hline 26 weeks. & 58.2 & 79.5 & 0.25 (0.10 to 0.63$)$ & $0.00^{* *}$ \\
\hline 52 weeks. & 69.1 & 69.9 & 0.93 (0.39 to 2.25$)$ & 0.88 \\
\hline
\end{tabular}

Values presented are model estimates of general linear mixed-effects models with a random intercept and adjusted for baseline and location. Regression coefficients can be interpreted as mean differences between interventions at a certain follow-up moment compared with baseline. Positive values favor the manual therapy group For Odds Ratios the reference group is physiotherapy

Abbreviations: ITT intention-to-treat, Cl confidence interval, GPE global perceived effect, NDI Neck Disability Index, NRS-P Numerical Rating Scale for Pain, OR odds ratio, MCID Minimal Clinical Important Difference 


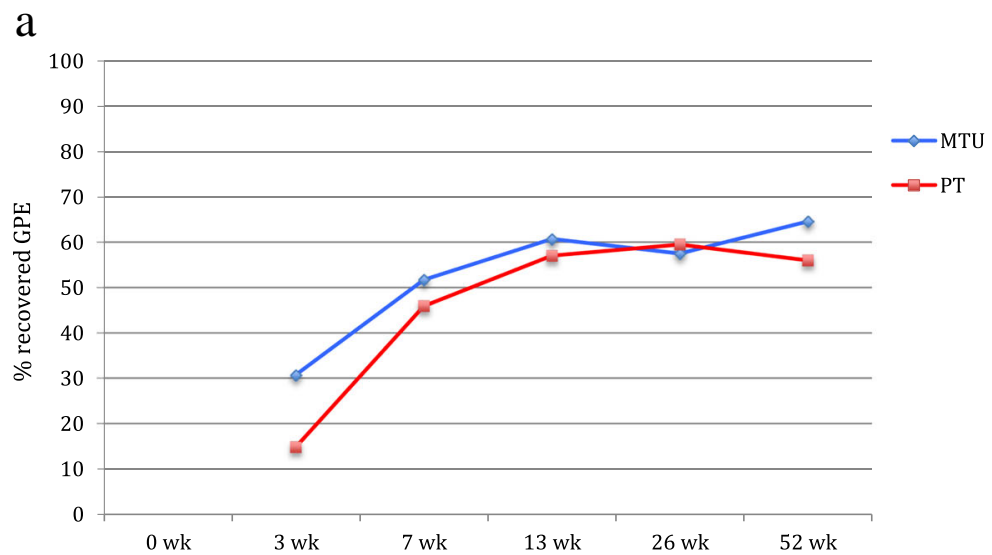

b
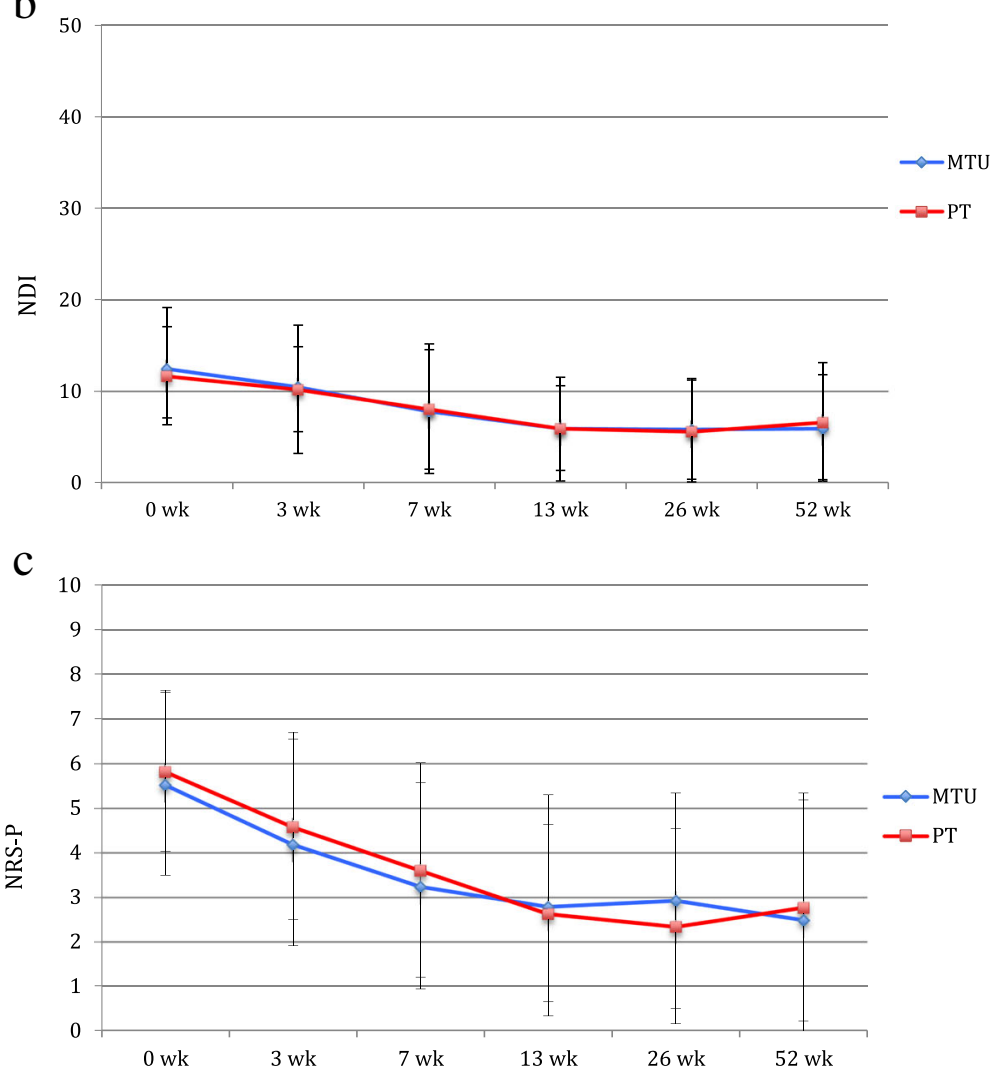

Fig. 2 Scores, with standard deviation bars, for manual therapy (MTU group; $n=90$ ) and physical therapy (PT group; $n=91$ ): a - GPE: Global Perceiver Effect (\% recovery), b - NDI: Neck Disability Index (0-50), c - NRS-P: Numeric Rating Scale for Pain (0-10). Abbreviations: GPE Global Perceived Effect, NDI Neck Disability Index, NRS-P Numeric Rating Scale for Pain, wk. weeks, MTU Manual Therapy Utrecht, PT Physical Therapy

Responder analysis is presented in Table 2. Overall, no statistically significant differences were found except for GPE at 3 weeks (adjusted odds ratio 3.97 , see above), and for pain at 26 weeks (NRS-P $\geq 2$ points) in favor of the PT group (80\%) versus the MTU group (58\%); adjusted odds ratio of 0.25 ( $95 \% \mathrm{CI}, 0.10$ to $0.63 ; P=0.00$ ).

The MTU group participated in a mean of $3.1(\mathrm{SD}=1.5$, range 0-8) treatment sessions during the intervention period versus $5.9(\mathrm{SD}=3.4$, range $0-20)$ for the PT group (7 weeks). Over the one-year follow-up period the number of treatments was 6.1 in the MTU group $(\mathrm{SD}=5.4$, range $0-33)$ versus $10.0(\mathrm{SD}=6.8$, range $0-29)$ in the PT group.

\section{Adverse events}

Minor, short-term adverse events were reported, but no serious complications occurred (Table 3). There were no significant differences in adverse events between the two 
Table 3 Adverse events for both treatments

\begin{tabular}{|c|c|c|c|c|c|c|c|c|c|}
\hline \multicolumn{2}{|l|}{ Adverse events } & \multicolumn{3}{|l|}{3 weeks } & \multicolumn{2}{|l|}{7 weeks } & \multicolumn{3}{|l|}{13 weeks } \\
\hline & MTU & PT & $p$ & MTU & PT & $p$ & MTU & PT & $p$ \\
\hline Number & 40 & 31 & 0.1723 & 31 & 23 & 0.1964 & 17 & 12 & 0.3179 \\
\hline Severity (NRS) & $4.8(3.9)$ & $5.3(2.6)$ & 0.3607 & $4.1(3.1)$ & $3.8(2.8)$ & 0.6456 & $4.9(3.0)$ & $3.6(2.8)$ & 0.2508 \\
\hline \multicolumn{10}{|l|}{ Time of onset (n) } \\
\hline$<30 \min$ & 4 & 7 & & 5 & 4 & & 3 & 0 & \\
\hline$>30$ min. $<4$ h. & 14 & 7 & & 5 & 4 & & 2 & 3 & \\
\hline$>4$ h., $<24$ h. & 11 & 8 & & 11 & 8 & & 3 & 1 & \\
\hline$>24 \mathrm{~h}$ & 7 & 6 & & 5 & 5 & & 6 & 1 & \\
\hline \multicolumn{10}{|l|}{ Duration (n) } \\
\hline$<10 \min$ & 0 & 0 & & 3 & 2 & & 4 & 1 & \\
\hline$>10$ min., $<1 \mathrm{~h}$. & 4 & 5 & & 1 & 2 & & 2 & 0 & \\
\hline$>1$ h., $<24$ h. & 13 & 9 & & 10 & 5 & & 8 & 3 & \\
\hline$>24 \mathrm{~h}$ & 20 & 13 & & 13 & 12 & & 4 & 2 & \\
\hline
\end{tabular}

Abbreviations: MTU Manual Therapy Utrecht, PT Physical Therapy, NRS Numeric Rating Scale, $n$ number, min minutes, $h r$ hours

groups in number $(n=40$ for MTU group and $n=31$ for PT group), severity (NRS-P 4.8 for MTU group and 5.4 for PT group) or duration (mostly less than $24 \mathrm{~h}$ ). The most frequently reported reactions were increased neck pain (17 out of 40 in MTU group and 14 out of 31 PT group), headache (3 of 40 in MTU group and 5 of 31 in PT group), back pain (7 of 40 in MTU group, 1 out of 31 in PT group), and dizziness and pins and needles in arms ( 2 in both groups).

\section{Patient preferences}

In both groups 20 patients preferred MTU. In the MTU group 9 patients preferred physical therapy, whereas 8 patients in the PT group preferred physical therapy. Other patients had no treatment preference. No differences on the mean CEQ scores were found between the MTU group and the PT group and between the patients preferring MTU or PT, compared to the non-preference patients. No significant differences in GPE, functioning (NDI) or pain (NRS-P) were found between patients who received their preferred intervention $(n=28)$ compared with those who did not receive their preferred treatment $(n=29)$.

\section{Discussion}

This pragmatic RCT in 181 patients with non-specific neck pain ( $>2$ weeks and $<1$ year) found no statistically significant overall differences in primary and secondary outcomes between the MTU group and PT group. The results at 7 weeks and 1 year showed no statistically and clinically significant differences. The assumption was that MTU was more effective based on the theoretical principles of mobilization of the chain of skeletal and movement-related joint functions of the spine, pelvis and extremities, and preferred movement pattern in the execution of a task or action by an individual, but that was not confirmed compared with standard care (PT).

\section{Adverse events}

Around $40 \%$ the patients in total reported various minor adverse events attributable to the treatment, following the classification terms for adverse events by Carnes et al. [48]. There were no significant differences between the MTU group and PT group in number, severity or duration of adverse events. RCTs are not the best research method for estimating the frequency of adverse events due to the recorded design of inclusion and treatment protocol. Large observational cohort studies might be expected to give a more accurate assessment [52].

\section{Attention bias and treatment sessions}

In this study, the mean number of treatment sessions in the MTU group was lower at 7 weeks and at 1 year. The initial maximum number of treatment sessions was 6 for MTU and 9 for PT; the time per session was 30-60 min and 30 min for MTU and PT, respectively. These parameters were described in the study protocol and are in line with standard Dutch clinical practice. Although the registration of the duration (number of minutes) of each treatment session was incomplete, the available data showed that only a minority $(10 \%)$ of patients in the MTU group was treated longer than $30 \mathrm{~min}$. So, overall the number of treatment sessions was higher in the PT group. However, as there were no differences in effects, we consider the risk of attention bias to be low.

\section{Cost-effectiveness}

A review of Michaleff et al. [53] supported the use of spinal manipulative therapy (SMT) in clinical practice as a cost-effective treatment when used alone or in combination with other treatment approaches. This review showed that while the effectiveness of SMT is comparable to other treatments, SMT is the more cost-effective 
treatment option. As Rubinstein et al. [54] have stated, because many treatments for spinal pain have comparable outcomes, distinctions in terms of cost-effectiveness should enjoy a high priority. An evaluation of the costeffectiveness of the present study was recently published [55]. In summary, the intervention costs and healthcare costs were significantly lower in the MTU group than in the PT group, whereas unpaid productivity costs were significantly higher. Total costs did not significantly differ between the MTU group and PT group. The conclusion was that MTU was not cost-effective in comparison with PT [55]. Consequently, the choice for MTU or PT can be left to the preferences of patients and care providers.

\section{Perspectives in relation to literature}

The patient population in our pragmatic study is comparable to populations described in general medical care. Additionally, the prevalence of co-morbidity is in line with the prevalence of co-morbidities for people visiting medical practices [56].

Our results are in line with the international literature on this subject, suggesting that SMT has a similar efficacy to other treatments [12, 57-60].

Present results are probably most comparable to two other Dutch studies by Hoving et al. [14, 15] and Pool et al. [61], given similar patient populations within the Dutch health care system. This is evident from the comparison of baseline scores (i.e. pain, function, duration of symptoms) with those studies [14, 15, 61]. Hoving et al. $[14,15]$ compared manual therapy to physical therapy and usual care (mainly based on a "wait and see" policy) provided by GPs. Manual therapy and PT were found to be more effective than GP care, with manual therapy superior to physical therapy, in contrast to our study. The reasons behind these differences of these studies may include slightly older participants in our study (mean age 50 years) compared to Hoving et al. and Pool et al (both mean age 45 years) $[14,15,61]$. A second difference was the higher success rate on GPE in the studies of Hoving et al. (72\% in MT group) $[14,15]$ and Pool et al. (70\% in MT group) [61], compared to this study (61\% in MTU group) at 13 weeks. This may have been due to the way data on outcomes were collected. In the study by Hoving et al. $[14,15]$ patients visited the research assistant and in the study by Pool et al. [61] a researcher collected patient data during a home visit. Because patients participating in research are known to try to meet an investigator's expectations [62], we eliminated personal contact between patients and research assistant through the use of web-based questionnaires and by avoiding face-to-face meetings or other personal contact during the follow-up period. A third explanation may be differences between the interventions compared to Hoving et al. [14, 15]. While the aim of PT treatment was comparable in both studies, Hoving et al. [14, 15] used manual therapy based on coordination and stabilization techniques (exercises) to treat segmental movement dysfunction of the spine, which might explain a better outcome for the MT group compared to the PT group.

The aim of this study was to evaluate the effectiveness of MTU as monotherapy and to compare MTU with PT, particularly exercise therapy. Both monotherapies are equally effective in patients with neck pain. It appears that clinicians are more and more in favor of using a broad-spectrum approach to treating patients with musculoskeletal pain, particularly neck pain and low back pain. Manual therapy is often combined with exercise therapy and pain education to treat patients with neck pain. Moderate quality evidence supports this combined therapy [63]. Studies of multimodality therapy should consider appropriate study designs, such as factorial designs.

\section{Pattern of responses to primary care treatments}

If the results of this study are placed in a broader perspective, a common trend in outcomes is visible, as shown in the pattern of treatments outcomes in patients with low back pain $[12,64]$. After an initial improvement in pain and functioning for both groups within 13 weeks, a slower reduction and stabilization of pain and functioning were followed in up to 52-week follow-up in both groups. This pattern of treatment outcome is quite similar in clinical trials with patients with low back pain unrelated to the type of treatment, particularly manual therapies It is plausible that such a pattern of responses to primary care treatments also occurs in clinical trials with patients with neck pain [12].

Artus et al. [64] provide several explanations for these comparable treatments outcomes in trials. Firstly, nonspecific factors, such as natural history and regression to the mean, non-specific effects of treatments (patients' and therapists' characteristics such as their beliefs, expectation, previous experiences and the attention given in the trial), differences of mean versus individual responses (averages in trials neutralizing individual variation) and the large overall response to treatment.

Besides these non-specific factors, there are interventionspecific factors. The treatment used in this trial was a manual therapeutic treatment per MTU that involves specific treatment ingredients based on arthrokinematic and osetokinematic principles of intra-articular joint movements of the spine, pelvis and extremities, as basis for promoting the individual preferred movement pattern.

With neck pain, many treatment options, particularly manual therapies, are available with often limited scientific evidence. Internationally, there are different schools of manual therapy. Some of these schools function as distinct professional groups (e.g., chiropractors, napropaths and osteopaths), while others consider themselves 
a specialization of physiotherapy (e.g., manual physical therapists). There is discussion about whether the treatment techniques used in these different schools contain essential differences and intervention-specific factors [18]. On the one hand, manual therapists, physiotherapists, chiropractors, napropaths, and osteopaths are educated in different theoretical assumptions and supposed underlying mechanisms, and on the other hand they use slightly different treatment techniques [18]. It has been suggested that the differences in practical applications of manual techniques are found mainly in amplitude and velocity of the mobilization and manipulation techniques [18]. However, it is questionable whether these schools are so different. There is room for discussion whether it is necessary and/or desirable emphasizing this diversity in manual therapies [65], considering that, despite the differences in approach (which are often small, subtle and theoretical in nature), there are no demonstrable differences in effectiveness [12].

\section{Limitations}

Some weaknesses in this study should be considered when interpreting our results. One issue deals with recruitment of patients, which started well but later slowed down. We therefore added an additional recruitment strategy using local newspapers. This could potentially have influenced the type or the severity of symptoms of patients at baseline $[66,67]$. It has been shown that the recruitment method affected the clinical characteristics (number of joints affected) and physical functioning (pain and tiredness) of patients recruited for a study of osteoarthritis of the hip or knee. However, a mix of recruitment strategies as used in our study should not affect treatment outcomes, on the condition that adjustments are made for differences at baseline [67-69]. The chosen strategy has no influence on the results.

A second issue worthwhile discussing is the partial overlap between the two treatment arms in this study. Although this is often unavoidable in a pragmatic trial, it may have resulted in bias. In both interventions patients received individualized advice with respect to activities of daily living and lifestyle and all received homework exercises. This may have decreased the contrast between the MTU group and the PT group, making this too small to lead to differences in outcomes.

A third potential limitation is the lack of a 'no-treatment' arm in this study. As it is not acceptable on ethical grounds to compare MTU with no treatment, because with no intervention patients are withheld a proved effective intervention. An indirect comparison with previous research in a similar setting is the most feasible approach to differentiate our results between regression to the mean and the natural history of the disease. Hoving et al. [14, 15] compared manual therapy and physical therapy with a 'wait and see' policy in Dutch primary care. The results of this study suggested that manual therapy and physical therapy are more effective than the "wait and see" policy. It seems reasonable to assume that the effects of MTU and PT identified in our study do not reflect the natural course or regression to the mean.

Another issue that needs to be taken into account is the mean baseline scores on NDI for both groups, which are low in our study, 12.5 in the MTU group and 11.7 in the PT group, equivalent to mild neck disability [70]. Kato et al. [71] determined the cut-off value of the NDI to detect neck pain associated with disability in a Japanese population to be 15 points. As this was a Japanese study, intercultural differences could play a role in the perceived limitations of activities. During follow-up, the NDI scores improved in both intervention groups. The mean scores at 7 weeks (7.9 points in both groups) and 13 weeks (5.9 points in both groups) were considered as 'no remaining disability' [70]. In this NDI range (around 12 of 50 points, $\mathrm{SD}+/-6$ ) statistically significant and clinically meaningful differences between groups are probably difficult to detect. The low baseline scores may explain why no clinically relevant and significant differences on the NDI scores were observed between the two groups, and there was potentially little room for improvement.

\section{Clinical implications}

The results of this study are that MTU and PT (active exercise therapy) do not differ in terms of effectiveness with both groups achieving similar improvements in pain and functioning. In shared decision-making, the choice of treatment options will be based on personal preferences of patient and therapist, with previous patient's and therapist's experience and the expected number of treatments to play a role.

\section{Conclusions}

Patients with non-specific neck pain improved in both groups without statistical significantly or clinically relevant differences between the MTU and PT groups during one-year follow-up.

\section{Abbreviations}

MTU: Manual Therapy Utrecht; PT: Physical therapy; GP: General practitioners; RCT: Randomized controlled trial; NRS: PNumeric rating scale for pain;

CEQ: Credibility/Expectancy Questionnaire; FABQ: Fear Avoidance Belief Questionnaire; GPE: Global perceived effect; NDI: Neck Disability Index; MCIC: Minimal clinically important change; ICF: International Classification of Functioning, Disability and Health; SMT: Spinal manipulative therapy

\section{Acknowledgements}

Not applicable.

Funding

This study was partially funded by Stichting Gezondheidszorg Spaarneland (Healthcare Foundation Spaarneland), The Netherlands. They had no role in designing, collecting or analyzing data, or in drafting the article. 


\section{Availability of data and materials}

The datasets during and/or analyzed during the current study are available from the corresponding author on reasonable request.

\section{Authors' contributions}

$R G, L V A, H K, H L, B C M S, R A B O, M W T$ were involved in the (conceptual) design; RG, LVA, HK, HL in data collection; RG, JM conducted the statistical analysis. All authors have read and approved the final manuscript.

\section{Competing interests}

$R G, L A, H K$ and $H L$ have a private MTU practice. There are no other competing interests.

\section{Consent for publication}

Not applicable.

\section{Ethics approval and consent to participate}

Ethical approval was obtained from the Medical Ethics committee CMO ArnhemNijmegen (NL21128.091.08).

\section{Publisher's Note}

Springer Nature remains neutral with regard to jurisdictional claims in published maps and institutional affiliations.

\section{Author details}

${ }^{1}$ Radboud University Nijmegen Medical Centre, Scientific Institute for Quality of Health Care, Geert Grooteplein 21, 6525 EX Nijmegen, The Netherlands. ${ }^{2}$ Department of Health Sciences, Faculty of Earth \& Life Sciences, Vrije Universiteit Amsterdam, Amsterdam Public Health research institute, De Boelelaan 1085, 1081 HV Amsterdam, The Netherlands. ${ }^{3}$ Avans+, University of Applied Science, Heerbaan 14-40, 4817 NL Breda, The Netherlands. ${ }^{4}$ Department of Health and Rehabilitation Sciences, University of Cape Town, Anzio Road 7935, Cape Town, South Africa. ${ }^{5}$ Department of Epidemiology and Biostatistics \& EMGO Institute for Health and Care Research, VU University Medical Centre, Amsterdam, The Netherlands. ${ }^{6}$ Department of Manual Therapy, Vrije Universiteit Brussel, Faculty of Medicine and Pharmacy, Brussels, Belgium.

\section{Received: 3 January 2017 Accepted: 5 April 2017}

\section{Published online: 28 April 2017}

\section{References}

1. Vos T, et al. Global, regional, and national incidence, prevalence, and years lived with disability for 310 diseases and injuries, 1990-2015: a systematic analysis for the Global Burden of Disease Study 2015. The Lancet. 2016;388: 1545-602.

2. Hoy DG, Protani M, De R, Buchbinder R. The epidemiology of neck pain Best Pract Res Clin Rheumatol. 2010;6:783-92.

3. Hogg-Johnson S, van der Velde G, Carroll $\sqcup$, Holm LW, Cassidy JD, Guzman J, Côté P, Haldeman S, Ammendolia C, Carragee E, Hurwitz E, Nordin M, Peloso P, Bone and Joint Decade 2000-2010 Task Force on Neck Pain and Its Associated Disorders. The burden and determinants of neck pain in the general population: results of the Bone and Joint Decade 2000-2010 Task Force on Neck Pain and Its Associated Disorders. Spine. 2008:33:39-51.

4. Guzman J, Hurwitz EL, Carroll L, Haldeman S, Côté P, Carragee EJ, Peloso PM, van der Velde G, Holm LW, Hogg-Johnson S, Nordin M, Cassidy JD, Bone and Joint Decade 2000-2010 Task Force on Neck Pain and Its Associated Disorders. A new conceptual model of neck pain: linking onset, course, and care: the Bone and Joint Decade 2000-2010 Task Force on Neck Pain and Its Associated Disorders. Spine. 2008;33:14-23.

5. Picavet HSJ, Schouten JSAG. Musculoskeletal pain in the Netherlands: prevalences, consequences and risk groups, the DMC3-study. Pain. 2003; 102:167-78.

6. Badcock $\amalg$, Lewis M, Hay EM, Croft PR. Consultation and the Outcome of Shoulder-Neck Pain: a Cohort Study in the Population. J Rheumatol. 2003; 30:2694-9.

7. Martin BI, Deyo RA, Mirza SK, Turner JA, Comstock BA, Hollingworth W, Sullivan SD. Expenditures and health status among adults with back and neck problems. JAMA. 2008;299:656-64.

8. Haldeman S, Carroll $\sqcup$, Cassidy JD, Bone and Joint Decade 2000-2010 Task Force on Neck Pain and Its Associated Disorders. he empowerment of people with neck pain: introduction: the Bone and Joint Decade 2000-2010 Task Force on Neck Pain and Its Associated Disorders. Spine. 2008:33:8-13.

9. Côté $P$, Kristman V, Vidmar M, Van Eerd D, Hogg-Johnson S, Beaton D, Smith PM. The prevalence and incidence of work absenteeism involving neck pain: a cohort of Ontario lost-time claimants. Spine. 2008;33:192-8.

10. Vos C, Verhagen A, Passchier J, Koes B. Management of acute neck pain in general practice: a prospective study. Br J Gen Pract. 2007;57:23-8.

11. Borghouts JA, Koes BW, Bouter LM. The clinical course and prognostic factors of non-specific neck pain: a systematic review. Pain. 1998;77:1-13.

12. Gross A, Langevin P, Burnie SJ, Bédard-Brochu MS, Empey B, Dugas E, FaberDobrescu M, Andres C, Graham N, Goldsmith CH, Brønfort G, Hoving JL, LeBlanc F. Manipulation and mobilisation for neck pain contrasted against an inactive control or another active treatment (Review). Cochrane Database Syst Rev. 2016;9:CD004249.

13. Gross AR, Paquin JP, Dupont G, Blanchette $S$, Lalonde $P$, Cristie T, Graham N, Kay TM, Burnie SJ, Gelley G, Goldsmith CH, Forget M, Santaguida PL, Yee AJ, Radisic GG, Hoving JL, Bronfort G, Groupo CO. Exercises for mechanical neck disorders: A Cochrane review update. Man Ther. 2016;24:25-45.

14. Hoving JL, Koes BW, de Vet HCW, van der Windt DAWM, Assendelft WJJ, Van Mameren H, Devillé WLJM, Pool JJM, Scholten RJPM, Bouter LM. Manual therapy, physical therapy, or continued care by a general practitioner for patients with neck pain. A randomized, controlled trial. Ann Intern Med. 2002;136:713-22.

15. Hoving JL, de Vet HCW, Koes BW, Van Mameren H, Devillé WLJM, van der Windt DAWM, Assendelft WJJ, Pool JJM, Scholten RJPM, Korthals-de Bos IBC, Bouter LM. Manual therapy, physical therapy, or continued care by the general practitioner for patients with neck pain: long-term results from a pragmatic randomized clinical trial. Clin J Pain. 2006;22:370-7.

16. Korthals-de Bos IBC, Hoving JL, van Tulder MW, Rutten-van Mölken MPMH, Adèr HJ, de Vet HCW, Koes BW, Vondeling H, Bouter LM. Cost effectiveness of physiotherapy, manual therapy, and general practitioner care for neck pain: economic evaluation alongside a randomised controlled trial. BMJ. 2003;326:911.

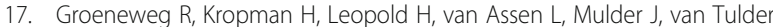
MW, Oostendorp RA. The effectiveness and cost-evaluation of manual therapy and physical therapy in patients with sub-acute and chronic non specific neck pain. Rationale and design of a Randomized Controlled Trial (RCT). BMC Musculoskelet Disord. 2010;11:14

18. van de Veen EA, de Vet HCW, Pool JJM, Schuller W, de Zoete A, Bouter LM. Variance in manual treatment of nonspecific low back pain between orthomanual physicians, manual therapists, and chiropractors. J Manipulative Physiol Ther. 2005;28:108-16.

19. Binder Al. Cervical spondylosis and neck pain. BMJ. 2007:334:527-31.

20. Hoving J. Prognostic factors for neck pain in general practice. Pain. 2004; 110:639-45.

21. Schellingerhout JM, Verhagen AP, Heymans MW, Pool JJM, Vonk F, Koes BW Wilhelmina de Vet HC. Which subgroups of patients with non-specific neck pain are more likely to benefit from spinal manipulation therapy, physiotherapy, or usual care? Pain. 2008;139:670-80.

22. van der Meulena MC, Huiskes R. Why mechanobiology? A survey article. J Biomech. 2002:35:401-14.

23. van der Bijl G. Het Individuele Functiemodel in de Manuele Therapie. De Tijdstroom: Lochem; 1986.

24. Van Wylick H, Oostendorp RAB, van der Zanden, Duquet W. Preferential movements: preference or dislike. Tijdschrift voor Manuele Geneeskunde 2004;2:16-21.

25. Maitland GD, English K, Banks K, Hengeveld E. Maitland's Vertebral Manipulation. 6 edition. Oxford: Butterworth-Heinemann; 2001.

26. Bronfort G, Haas M, Evans R, Leiniger B, Triano J. Effectiveness of manual therapies: the UK evidence report. Chiropr Osteopat. 2010;18:3.

27. Kay TM, Gross A, Goldsmith CH, Rutherford S, Voth S, Hoving JL, Bronfort G, Santaguida PL. Exercises for mechanical neck disorders. Cochrane Database Syst Rev. 2012:8:CD004250

28. Jensen I, Harms-Ringdahl K. Neck pain. Best Pract Res Clin Rheumatol. 2007; 21:93-108.

29. Devilly GJ, Borkovec TD. Psychometric properties of the credibility/expectancy questionnaire. J Behav Ther Exp Psychiatry. 2000;31:73-86.

30. Smeets RJEM, Beelen S, Goossens MEJB, Schouten EGW, Knottnerus JA, Vlaeyen JWS. Treatment expectancy and credibility are associated with the outcome of both physical and cognitive-behavioral treatment in chronic low back pain. Clin J Pain. 2008;24:305-15. 
31. Boersma K, Linton SJ. Expectancy, fear and pain in the prediction of chronic pain and disability: A prospective analysis. Eur J Pain. 2006;10:551.

32. Vendrig A, Deutz P, Vink I. Nederlandse Vertaling en Bewerking Van De FearAvoidance Beliefs Questionnaire. Ned Tijdschrift voor Pijn en Pijnbestrijding. 1998;18:11-4.

33. Landers MR, Creger RV, Baker CV, Stutelberg KS. The use of fear-avoidance beliefs and nonorganic signs in predicting prolonged disability in patients with neck pain. Man Ther. 2008;13:239-48.

34. Chiu TW. Effects of fear-avoidance beliefs on Chinese patients with neck pain. Hong Kong Med J. 2009;15:S9-12.

35. Nederhand MJ, ljzerman MJ, Hermens HJ, Turk DC, Zilvold G. Predictive value of fear avoidance in developing chronic neck pain disability: consequences for clinical decision making. Arch Phys Med Rehabil. 2004;85:496-501.

36. Cai C, Ming G, Ng LY. Development of a clinical prediction rule to identify patients with neck pain who are likely to benefit from home-based mechanical cervical traction. Eur Spine J. 2011;20:912-22.

37. Aaronson N, Muller M, Cohen P, Essink-Bot M, Fekkes M, Sanderman R, Sprangers M, te Velde A, Verrips E. Translation, validation, and norming of the Dutch Language Version of the SF-36 health survey in community and chronic disease populations. J Clin Epidemiol. 1998;51:1055-68.

38. van der Roer N, Ostelo RWJG, Bekkering GE, van Tulder MW, de Vet HCW. Minimal clinically important change for pain intensity, functional status, and general health status in patients with nonspecific low back pain. Spine. 2006;31:578-82

39. Vet HCW, Ostelo RWJG, Terwee CB, Roer N, Knol DL, Beckerman H, Boers M, Bouter LM. Minimally important change determined by a visual method integrating an anchor-based and a distribution-based approach. Qual Life Res. 2007;16:131-42.

40. Kamper SJ, Ostelo RWJG, Knol DL, Maher CG, de Vet HCW, Hancock MJ. Global Perceived Effect scales provided reliable assessments of health transition in people with musculoskeletal disorders, but ratings are strongly influenced by current status. J Clin Epidemiol. 2010;63:760-6. e1.

41. Jorritsma W, Vries GE, Dijkstra PU, Geertzen JHB, Reneman MF. Neck Pain and Disability Scale and Neck Disability Index: validity of Dutch language versions. Eur Spine J. 2011;21:93-100.

42. Jorritsma W, Dijkstra PU, Vries GE, Geertzen JHB, Reneman MF. Detecting relevant changes and responsiveness of Neck Pain and Disability Scale and Neck Disability Index. Eur Spine J 2012, DOI 10.1007/s00586-012-2407-8.

43. Vos CJ, Verhagen AP, Koes BW. Reliability and responsiveness of the Dutch version of the Neck Disability Index in patients with acute neck pain in general practice. Eur Spine J. 2006;15:1729-36.

44. Pool JJM, Ostelo RWJG, Hoving JL, Bouter LM, de Vet HCW. Minimal clinically important change of the Neck Disability Index and the Numerical Rating Scale for patients with neck pain. Spine. 2007;32:3047-51.

45. World Health Organization. Towards a Common Language for Functioning, Disability and Health ICF. WHO/EIP/GPE/CAS/01.3. 2002.

46. Rosier EM, ladarola MJ, Coghill RC. Reproducibility of pain measurement and pain perception. Pain. 2002;98:205-16

47. Kovacs FM, Abraira V, Royuela A, Corcoll J, Alegre L, Tomas M, Mir MA, Cano A, Muriel A, Zamora J, Gil Del Real MT, Gestoso M, Mufraggi N. Minimum detectable and minimal clinically important changes for pain in patients with nonspecific neck pain. BMC Musculoskelet Disord. 2008:9:43

48. Carnes D, Mullinger B, Underwood M. Defining adverse events in manual therapies: A modified Delphi consensus study. Man Ther. 2010;15:2-6.

49. Pool JJM, Ostelo RWJG, Köke AJ, Bouter LM, de Vet HCW. Comparison of the effectiveness of a behavioural graded activity program and manual therapy in patients with sub-acute neck pain: Design of a randomized clinical trial. Man Ther. 2006;11:297-305

50. Twisk JW, de Vente W. Attrition in Longitudinal Studies. How to Deal with Missing Data. J Epidemol 2002;55:329-337.

51. PEDro. https://www.pedro.org.au/english/downloads/pedro-scale/. Accessed Feb 2017.

52. Carnes D, Mars TS, Mullinger B, Froud R, Underwood M. Adverse events and manual therapy: A systematic review. Man Ther. 2010;15:355-63.

53. Michaleff ZA, Lin CWC, Maher CG, van Tulder MW. Spinal manipulationepidemiology: Systematic review of cost effectiveness studies. J Electromyogr Kinesiol 2012;22:655-62.

54. Rubinstein SM, van Middelkoop M, Assendelft WJ, de Boer MR, van Tulder MW. Spinal manipulative therapy for chronic low-back pain. Cochrane Database Syst Rev. 2011;2:CD008112.
55. van Dongen JM, Groeneweg R, Rubinstein SM, Bosmans JE, Oostendorp RAB, Ostelo RWJG, van Tulder MW. Cost-effectiveness of manual therapy versus physiotherapy in patients with sub-acute and chronic neck pain: a randomised controlled trial. Eur Spine J. 2016;25:2087-96.

56. Barnett K, Mercer SW, Norbury M, Watt G, Wyke S, Guthrie B. Epidemiology of multimorbidity and implications for health care, research, and medical education: a cross-sectional study. The Lancet. 2012;380:37-43.

57. van der Velde G, Hogg-Johnson S, Bayoumi AM, Cassidy JD, Côté P, Boyle E, Llewellyn-Thomas H, Chan S, Subrata P, Hoving JL, Hurwitz E, Bombardier C, Krahn $\mathrm{M}$. Identifying the best treatment among common nonsurgical neck pain treatments: a decision analysis. Spine. 2008;33:S184-91.

58. Wong JJ, Shearer HM, Mior S, Jacobs C, Côté P, Randhawa K, MSc HYM, Southerst D, Varatharajan S, Sutton D, van der Velde G, Carroll L, Ameis A, Ammendolia C, Brison R, Nordin M, Taylor-Vaisey A. Are manual therapies, passive physical modalities, or acupuncture effective for the management of patients with whiplash-associated disorders or neck pain and associated disorders? An update of the Bone and Joint Decade Task Force on Neck Pain and Its Associated Disorders by the OPTIMa collaboration. Spine J. 2016;16:1598-630

59. Vernon $\mathrm{H}$, Humphreys BK. Manual therapy for neck pain: an overview of randomized clinical trials and systematic reviews. Eura Medicophys. 2007;43: 91-118.

60. Hurwitz EL, Carragee EJ, van der Velde G, Carroll L, Nordin M, Guzman J, Peloso PM, Holm LW, Côté P, Hogg-Johnson S, Cassidy JD, Haldeman S, Bone and Joint Decade 2000-2010 Task Force on Neck Pain and Its Associated Disorders. Treatment of neck pain: noninvasive interventions: results of the Bone and Joint Decade 2000-2010 Task Force on Neck Pain and Its Associated Disorders. Spine. 2008:33:S123-52.

61. Pool JJM, Ostelo RWJG, Knol DL, Vlaeyen JWS, Bouter LM, de Vet HCW. Is a behavioral graded activity program more effective than manual therapy in patients with subacute neck pain? Results of a randomized clinical trial. Spine. 2010;35:1017-24.

62. Berthelot J-M, Le Goff B, Maugars Y. The Hawthorne effect: Stronger than the placebo effect? Joint Bone Spine. 2011;78:335-6.

63. Miller J, Gross A, D'Sylva J, Burnie SJ, Goldsmith CH, Graham N, Haines T, Bronfort $\mathrm{G}$, Hoving JL. Manual therapy and exercise for neck pain: A systematic review. Man Ther. 2010;15:334-54.

64. Artus M, van der Windt DA, Jordan KP, Hay EM. Low back pain symptoms show a similar pattern of improvement following a wide range of primary care treatments: a systematic review of randomized clinical trials. Rheumatology. 2010;49:2346-56.

65. Haldeman S, Dagenais S. A supermarket approach to the evidence-informed management of chronic low back pain. Spine J. 2008:8:1-7.

66. Holdsworth L, Webster V, McFadyen A. The Scottish Physiotherapy Self Referral Study Group. Are Patients Who Refer Themselves to Physiotherapy Different From Those Referred by GP's? Results of a National Trial. Physiotherapy. 2006;92:26-33

67. Veenhof C, Dekker J, Bijlsma JWJ, Van Den Ende CHM. Influence of various recruitment strategies on the study population and outcome of a randomized controlled trial involving patients with osteoarthritis of the hip or knee. Arthritis Rheum. 2005;53:375-82.

68. Geraets JJXR, de Groot IJM, Goossens MEJB, de Bruijn CPC, De Bie RA, van den Heuvel WJA, Dinant GJ. Comparison of two recruitment strategies for patients with chronic shoulder complaints. Br J Gen Pract. 2006;56:127-33.

69. Sherman KJ, Hawkes RJ, Ichikawa L, Cherkin DC, Deyo RA, Avins AL, Khalsa PS. Comparing recruitment strategies in a study of acupuncture for chronic back pain. BMC Med Res Methodol. 2009;9:69.

70. Vernon H. The Neck Disability Index: state-of-the-art, 1991-2008. J Manipulative Physiol Ther. 2008:31:491-502.

71. Kato S, Takeshita K, Matsudaira K, Tonosu J, Hara N, Chikuda H. Normative score and cut-off value of the Neck Disability Index. J Orthop Sci 2012, doi 10.1007/s00776-012-0276-y. 